\title{
Robotic Thyroid Surgery: Current Perspectives and Future Considerations
}

\author{
Patrick Aidan $^{a} \quad$ Asit Arora $^{b} \quad$ Balazs Lorincz ${ }^{c} \quad$ Neil Tolley ${ }^{d}$ \\ George Garas d, e \\ ${ }^{a}$ Department of Otorhinolaryngology and Head and Neck Surgery, American Hospital \\ of Paris, Paris, France; ${ }^{b}$ Department of Otorhinolaryngology and Head and Neck \\ Surgery, Guy's and St. Thomas' NHS Foundation Trust, London, UK; ' $D$ Department of \\ Otorhinolaryngology and Head and Neck Surgery, University Medical Centre \\ Hamburg-Eppendorf, Hamburg, Germany; ${ }^{d}$ Department of Otorhinolaryngology, Head \\ and Neck Surgery, St. Mary's Hospital, Imperial College Healthcare NHS Trust, London, UK; \\ e Department of Surgical Research and Innovation, Royal College of Surgeons of England, \\ London, UK
}

\section{Keywords}

Robotic surgery · Thyroid · Axillary approach · Retroauricular approach · Facelift · Transoral route $\cdot$ Evidence $\cdot$ Safety $\cdot$ Cosmesis $\cdot$ Policy

\begin{abstract}
Robotic transaxillary thyroidectomy, pioneered in South Korea, is firmly established throughout the Far East but remains controversial in Western practice. This relates to important population differences (anthropometry and culture) compounded by the smaller mean size of thyroid nodules operated on in South Korea due to a national thyroid cancer screening programme. There is now level 2 evidence (including from Western World centres) to support the safety, feasibility, and equivalence of the robotic approach to its open counterpart in terms of recurrent laryngeal nerve injury, hypoparathyroidism, haemorrhage, and oncological outcomes for differentiated thyroid cancer. Moreover, robotic thyroidectomy has been shown to be superior to open surgery for certain patient-reported outcome measures, namely scar cosmesis and pain. Downsides include its high cost, longer operative time, and risk of complications not encountered in open thyroidectomy (brachial plexus neurapraxia). Careful patient selection is paramount as this procedure is not for every patient, surgeon, or hospital. It should only be undertaken by high-volume surgeons operating as part of a multidisciplinary robotic team in specialised centres. Novel robotic approaches utilising the retroauricular and transoral routes for thyroidectomy have recently been described but further studies are required to establish their respective role in modern thyroid surgery.


Aidan et al.: Robotic Thyroid Surgery

\section{Introduction}

Although firmly established in the Far East, robotic transaxillary thyroidectomy remains a controversial technique in Western practice. The larger body habitus of Western patients adding to the technical difficulties of the procedure, cost, training, and ethical considerations have acted as a negative influence on adoption. At present, the technique has a niche role in patients with suitable pathology combined with a history of keloid and hypertrophic scarring or in those health care systems where personalised medicine has a role. In the Western World, the number of robotic procedures after initial enthusiasm has now diminished and reached a plateau. Surprisingly, the highest numbers are performed in low-volume institutions, which support personalised medicine to be a dominant driver $[1,2]$. In the early adoption phase, concerns were soon raised about unique complications associated with the procedure resulting in the publication of an American Thyroid Association consensus and guideline document [3].

A reflection and discussion on where robotic thyroidectomy presently resides on Rogers' [4] diffusion of innovations curve and the reasons for this are discussed in this review. The evidence for effectiveness and its value are also presented, the latter having relevance to its adoption in health care systems where resources are limited. Finally, some outline recommendations and conclusions are presented as to how the profession can move forward in evaluating and adopting new surgical technologies that may confer value in health care.

\section{History and Evolution of the Technique}

Thyroid nodules, including low-risk papillary thyroid cancer, are most commonly encountered in young women [5]. Naturally, this select patient group is the one most concerned about cosmesis. This is more so in the Far East where a horizontal neck scar is perceived to denote death [6].

The above concerns led to the development of "scarless" (in the neck) endoscopic thyroidectomy. A variety of routes have been described over the years including infraclavicular, breast, axillary, axilla-bilateral-breast, bilateral axilla-breast endoscopic approaches, and postauricular and axillary endoscopic approaches [7]. However, the multitude of endoscopic techniques trialled revealed none to be superior, all characterised by the same limitations of endoscopic surgery; namely the 2-dimensional visualisation, restrictions in instrument movement, fulcrum effect, need for insufflation, and dependence on multiple assistants [8].

Robotic technology with its unique features including a dual-channel stereoscopic endoscope offering simultaneous image magnification and depth perception through 3-dimensional visualisation combined with its wristed robotic instruments possessing 7 degrees of freedom substantially enhances surgical dexterity and was introduced to overcome the limitations characterising endoscopic surgery [9].

In 2009, the first large series $(n=100)$ of transaxillary gasless robotic thyroidectomy was published in South Korea [10]. The procedure proved to be popular among the local population, and its uptake was rapid in the Far East with thousands of cases performed over the subsequent years [11-13]. However, the uptake in the Western World has been significantly lower. Various factors have been implicated including differences in culture, anthropometry, remuneration issues, as well as the presence of a national thyroid cancer screening programme in South Korea leading to earlier detection of thyroid nodules when these are much smaller (often less than $1 \mathrm{~cm}$ in diameter) and thus easier to resect (Fig. 1) [1]. 
Aidan et al.: Robotic Thyroid Surgery

Fig. 1. Barriers to the uptake of robotic thyroidectomy in the Western World (as opposed to South Korea).

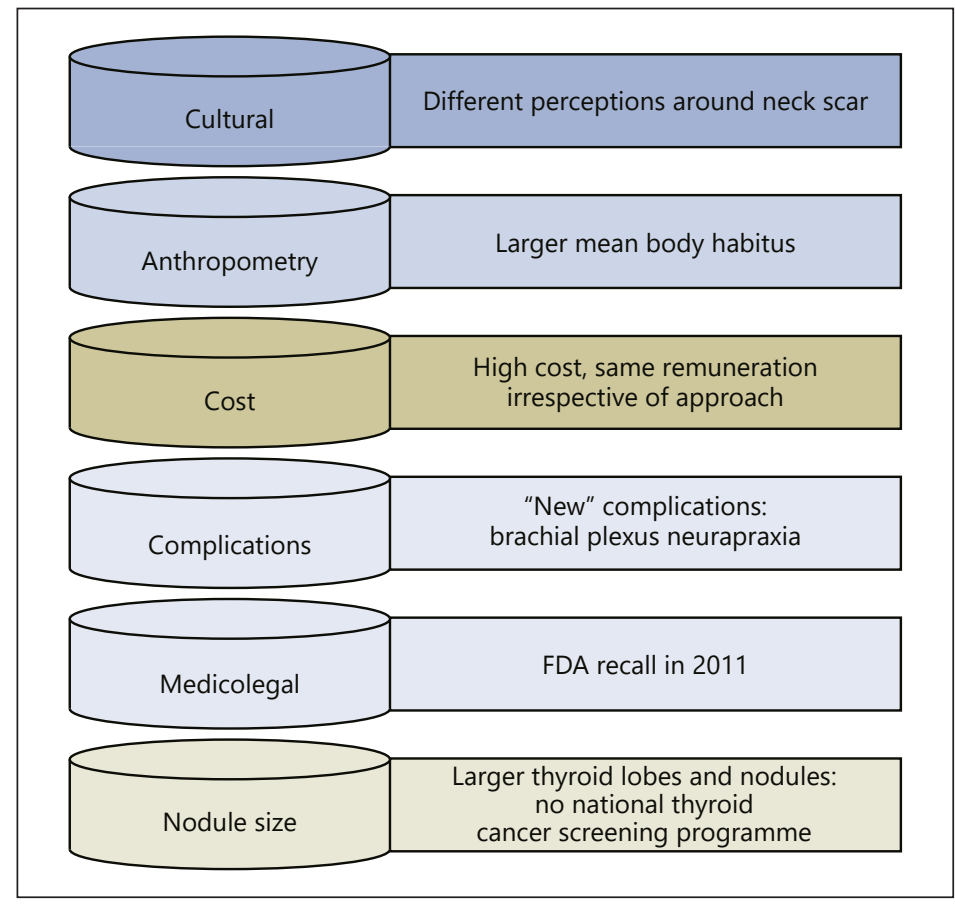

\section{The Evidence Base}

In today's practice, evidence-based surgery is paramount and as such, no discussion would be complete without presenting this [14].

Concerning feasibility, safety and cosmesis, there is level 2 a evidence supporting robotic thyroidectomy as a feasible and safe approach, cosmetically superior to open surgery. The feasibility and safety of robotic thyroidectomy are not limited to studies from the Far East (where the majority originate), but also from high-volume centres in the UK, Europe, and North America [1, 15-17].

Despite a 3- to 4-fold increase in the area of subcutaneous dissection required (Fig. 2) and an increased operative time, the literature has consistently shown that improvement of pain and paraesthesia following transaxillary robotic thyroid surgery is superior to conventional open surgery (level 2b) [1,18-20].

Five meta-analyses of cohort studies collectively pooling data from several thousand patients have evaluated the commonest complications relating to thyroid surgery, namely recurrent laryngeal nerve injury, hypoparathyroidism, and haemorrhage. No significant differences between robotic and open thyroidectomy in terms of transient and permanent recurrent laryngeal nerve injury, permanent hypoparathyroidism, or haemorrhage were identified (level 2a) [16, 21-24]. A higher incidence of transient hypoparathyroidism was detected in robotic thyroidectomy patients (level 2a) [16, 21].

Reviews in 2016 have extended this analysis to data from over 15 studies which supported the conclusion that robotic compared to conventional thyroidectomy conferred no difference relating to length of hospital stay, quality of life, wound infection, seroma, chyle leak, and tracheal injury (level 2a). On the other hand, early swallowing and scar satisfaction showed a significant benefit consistently favouring the robotic approach (level 2a) [21, 25].

In terms of oncological efficacy, there are several recent systematic reviews and metaanalyses pooling data from studies collectively reporting on several thousand cases that illus- 
Fig. 2. The area of subcutaneous dissection for robotic (red) and open (green) thyroidectomy when the patient is in the anatomical position.

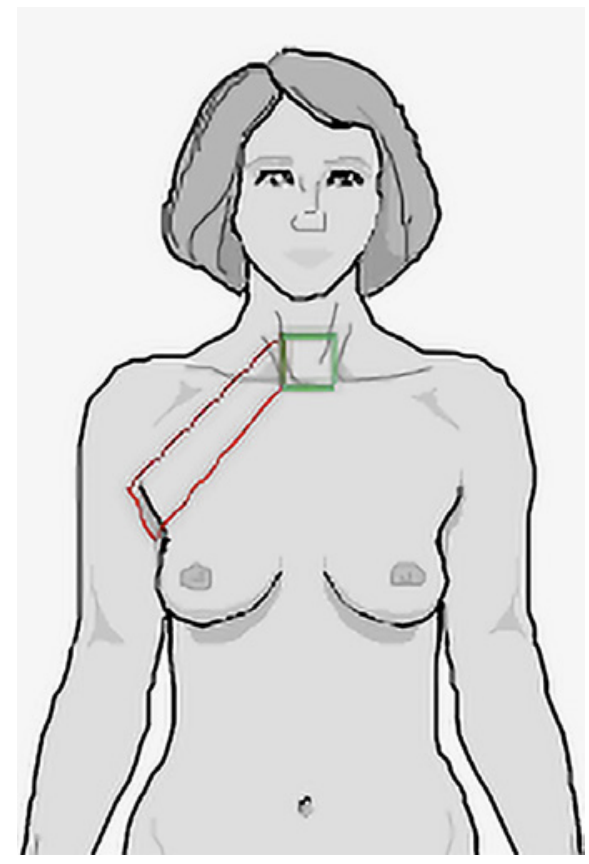

Fig. 3. Intraoperative robotic console image of the right thyroid lobe following entrance into the neck through the natural dehiscence of the sternal and clavicular heads of the sternocleidomastoid and retraction of the strap muscles.

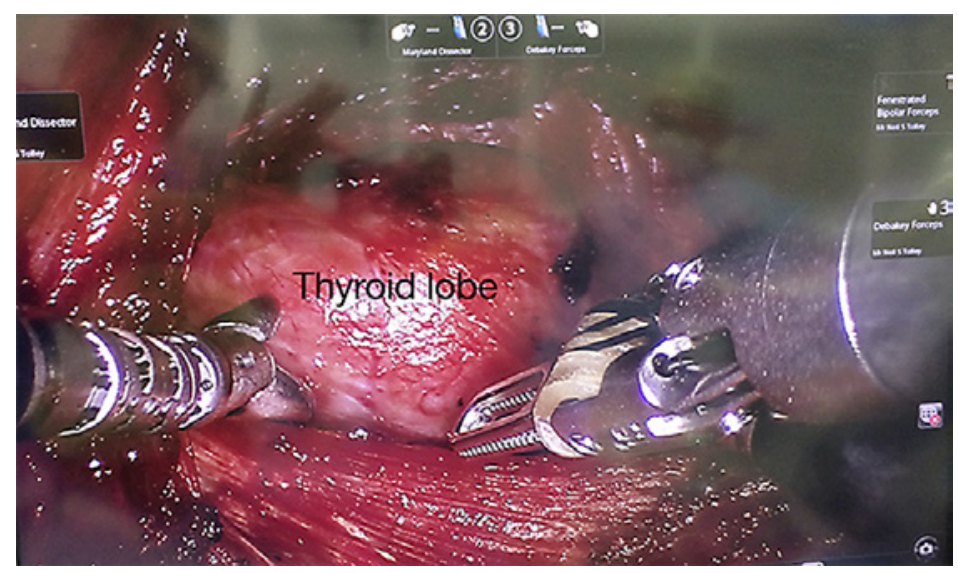

trate that there is no significant difference between the robotic and open approaches in terms of serum thyroglobulin levels, post-ablation radioactive iodine scan uptake, lymph node yield, locoregional recurrence and survival rates, thus confirming the oncological value of robotic thyroidectomy for differentiated thyroid cancer (level 2a) [13, 21, 25, 26].

Both short- (thyroglobulin) and long-term (locoregional recurrence and disease-specific survival) results have supported the safety and efficacy of robotic thyroidectomy in the treatment of thyroid cancer [11]. One large multi-institutional study evaluated a cohort of over a thousand patients again supporting its safety (level 2a) [27].

\section{Benefits}

As discussed, there is now level 2 evidence to support transaxillary robotic thyroidectomy for the treatment of benign and malignant disease in appropriately selected patients. Results are equivalent to the open approach. 
Fig. 4. Intraoperative robotic console image illustrating medial retraction of the thyroid lobe to expose the recurrent laryngeal nerve (circled) in the tracheooesophageal groove during robotic thyroidectomy.

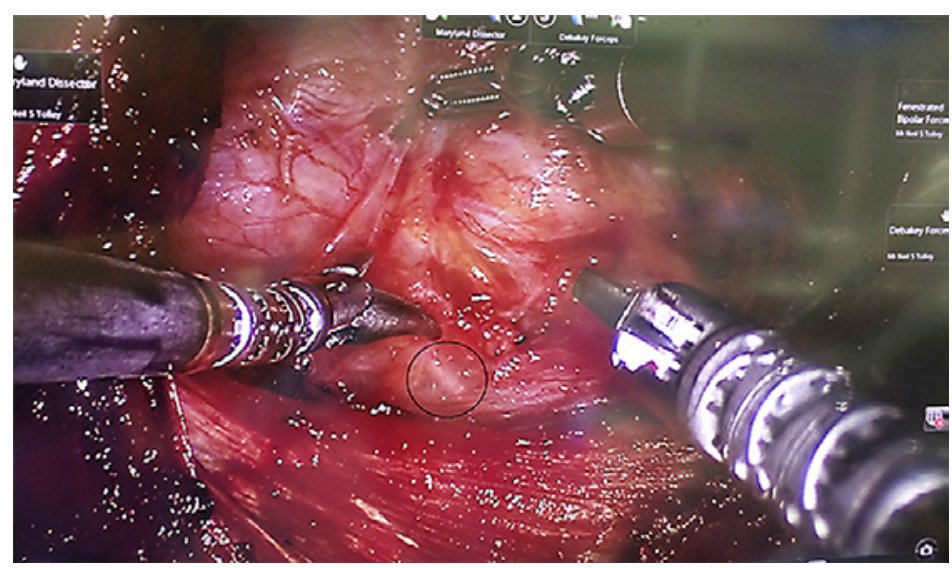

Moreover, robotic thyroidectomy confers superior cosmesis over the open approach. The evidence for this is level $1 \mathrm{~b}$ as in addition to the several case control and cohort studies evaluating patient-reported outcome measures $[1,28-30]$, there is also a randomised controlled trial to support this though the latter failed to demonstrate cosmetic superiority of the transaxillary robotic approach over minimally invasive video-assisted thyroidectomy [17].

There is more to robotic thyroid surgery than the avoidance of a neck scar [31, 32]. The improved cosmesis achieved by all extracervical thyroidectomy approaches should be viewed as a "bonus" for the patient, and never as the sole reason for offering this approach. Patient selection is paramount as discussed below [28].

A further benefit of robotic thyroid surgery is the combination of superior visualisation and precise tissue manipulation leading to potentially safer and more precise dissection (Fig. 3, 4) [33]. It is important to emphasise that training and proctorship have an important role in achieving the safe implementation and development of skills.

A 3-dimensional, magnified view, wristed robotic arms with 7 degrees of freedom, tremor filtering, and motion scaling are collectively exclusive to the da Vinci ${ }^{\circledR}$ surgical robot (Intuitive Surgical Inc., Sunnyvale, CA, USA) [34]. These collectively unique qualities facilitate the precise identification and handling of vital structures in the confined space of the neck [35].

\section{Drawbacks}

The prime drawback relates to the potential of brachial plexus injury, a "new" and unique complication of transaxillary robotic thyroid surgery. Despite its low incidence of $0.2 \%$, brachial plexus injury is nonetheless a very significant and worrying complication that patients should be informed of prior to seeking informed consent. Fortunately, not only is it rare, but also transient, being associated with the "traditional" transaxillary approach [36, 37]. The risk has now been considerably reduced since arm positioning was modified from the original technique described by Chung et al. [8] to the "extended salute position" $[1,26]$.

Then comes the issue relating to the longer operative time. Though it is a well-established fact that robotic thyroidectomy - even after plateauing of the surgeon's learning curve - adds to the operation around $45 \mathrm{~min}$ (39-54 min depending on the study), this has not been shown to bear any impact on patient outcomes, quality of life, or length of hospital stay.

Another drawback relates to the extent of subcutaneous dissection required for accessing the neck. Despite the area of the flap needed to be raised being 3-4 times that for the cervical 
Aidan et al.: Robotic Thyroid Surgery

approach (leading to the increased operative time along with the time needed for docking the robot), this increased area of dissection does not translate to increased pain and/or paraesthesia (level 2b) [1, 18-20].

Finally, tracheal and oesophageal injuries as well as chyle leaks have been reported with the transaxillary robotic approach. However, these complications are exquisitely rare and their incidence no higher than with open thyroidectomy [32].

\section{Cost-Effectiveness and Value}

The largest barrier to adoption of robotic thyroidectomy on a world stage is its prohibitive cost [38]. The cost of purchasing the da Vinci ${ }^{\circledR}$ surgical robot is GBP 1.5 million. Added to this are maintenance averaging GBP 120,000 per annum and surgical consumables at approximately GBP 1,300 per case [14]. Higher costs are achieved by the monopoly status of Intuitive Surgical Inc. in the surgical robotics market. Intuitive Surgical Inc. have employed aggressive business strategies including acquisition of key competitors (e.g. Computer Motion Inc., Santa Barbara, CA, USA) and patenting of several robotic components [39].

However, with the imminent entry of multinational medical device companies such as Medtronic Inc. (Minneapolis, MN, USA), Medrobotics Inc. (Raynham, MA, USA), and the Johnson \& Johnson Inc. (New Brunswick, NJ, USA) - Google Inc. (Mountain View, CA, USA) partnership in the surgical robotics arena, competition can be expected to drive down costs making robotic surgical technology more widely affordable [40].

By bringing down the costs incurred by the health care system, robotic thyroidectomy may in the future become cost-effective (or at least cost-neutral) [41, 42]. However, based on current prices, robotic thyroidectomy cannot qualify as a cost-effective intervention [43]. As an example, the National Institute for Health and Care Excellence in the UK loosely applies a cost-effectiveness threshold of GBP 30,000 per quality-adjusted life year in cases where efficacy is proven and GBP 20,000 per quality-adjusted life year in cases where clinical effectiveness is more controversial [44]. Formal cost-effectiveness studies are required to determine the true value of robotic thyroidectomy and inform policy. At this point, it is important to state that adoption of a new health care intervention is not purely an economic decision. Social, political, ethical, and moral considerations also need to be taken into account [45].

Finally, by centralising robotic thyroidectomy services to national high-volume centres and surgeons, further cost reduction can be achieved. This is through a combination of economies of scale and a decrease in operative time - the other main driver of cost - as surgeons surpass their learning curve [46, 47]. Centralisation will further enhance its value as in addition to the lower cost, outcomes have been shown to be consistently superior when robotic thyroid surgery is undertaken in specialised, high-volume centres [5, 48, 49].

\section{Current Status, Novel Approaches, and Future Role}

In less than a decade since the technique was pioneered in South Korea in 2009 [10], and despite numerous setbacks, the most significant being a medical device class II recall by the Food and Drug Administration in 2011 [50], robotic thyroidectomy has adapted and matured as a surgical technology [2].

Problems initially encountered with robotic thyroid surgery mostly related to the fact that the technique was originally developed for patients in the Far East, a substantially different cohort to Western patients in several aspects [1]. Early adoption within the Western 
Aidan et al.: Robotic Thyroid Surgery

World was made without considering and accounting for important population differences especially those relating to body size and cultural perceptions. In addition, the mean thyroid nodule size operated on in the Western World is significantly larger compared to that in South Korea due to the presence of a national thyroid cancer screening programme in the latter [1].

As a consequence and out of necessity, modifications were introduced to address these problems. These included alteration in arm positioning to reduce the potential for brachial plexus morbidity, the design of new retractors specifically for Western patients' body habitus (e.g. Imperial ${ }^{\circledR}$ or MODENA ${ }^{\circledR}$ retractor systems), and more careful patient selection [1]. As with all surgical techniques, the latter is of special importance.

More recently, the retroauricular (facelift) robotic approach has been described [51]. First performed in 2011 in the USA, this is likely to represent a more appropriate access route for Western patients due to the aforementioned anthropometric and mean nodule size differences compared to South Korean patients [51]. Advantages over the transaxillary approach include the reduced flap area (38\% less) and the obviation of the risk of brachial plexus injury [7]. Though considered a "more natural" approach, the evidence for retroauricular robotic thyroidectomy is level 3 at present with a small number of studies to date and an associated limited uptake of the technique [7]. Larger, comparative studies are needed to establish its role in modern thyroid surgery.

The latest development in the field relates to transoral robotic thyroidectomy [52]. Following animal and cadaveric studies, this procedure was first performed in live humans in 2016, and its safety and feasibility were demonstrated in both American and South Korean patients [52]. Though it is too early to appreciate its role in modern thyroid surgery, it is important to state that transoral robotic thyroidectomy represents the only "truly scarless" robotic thyroidectomy approach and the first application of natural orifice transluminal endoscopic surgery in thyroid surgery [7].

Robotic thyroidectomy (including all described approaches) is a complex and technically demanding operation. Intensive training and proctorship are required for its safe implementation combined with careful patient selection. Long-term outcomes are starting to emerge in the literature and predict a promising future for robotic thyroidectomy.

\section{Conclusion}

At present, robotic thyroidectomy is not for every patient, surgeon or hospital. Considered and measured patient selection is paramount. As with the adoption of all new surgical technology, scientific study and careful systematic evaluation should always be employed in addition to consideration of ethics. Evidence should guide the adoption of innovation, and commercial companies should financially support prospective ethically approved studies. Such studies will provide both the guidance and level of governance required for the safe and effective adoption of new surgical technology.

The evidence exists to support robotic transaxillary thyroidectomy for the treatment of benign and malignant disease in appropriately selected patients. This procedure should be undertaken by high-volume surgeons operating as part of a multidisciplinary robotic team in specialised centres. Centralisation will provide the infrastructure to ensure patient safety by audit and governance, facilitate and promote further research, and provide a platform for education and teaching. Novel robotic approaches utilising the retroauricular and transoral routes for thyroidectomy have recently been described but further studies are required to establish their respective role in modern thyroid surgery. 
Aidan et al.: Robotic Thyroid Surgery

\section{Disclosure Statement}

Dr. George Garas, MD, FRCS, holds a Royal College of Surgeons of England Doctoral Research Fellowship (Grant No. GG 1037600/2017-2018) and is also supported by Imperial College London (Grant No. CID 337755/2015-2018) and the Alexander S. Onassis Public Benefit Foundation (Grant No. F ZM 014-1/20162017). The funders had no role in the study design, data collection and analysis, decision to publish, or preparation of the manuscript. All authors declare no conflicts of any commercial interest.

\section{References}

- 1 Arora A, et al: Comparing transaxillary robotic thyroidectomy with conventional surgery in a UK population: a case control study. Int J Surg 2016;27:110-117.

2 Hinson AM, et al: Trends in robotic thyroid surgery in the United States from 2009 through 2013. Thyroid 2015;25:919-926.

3 American Thyroid Association Surgical Affairs Committee: American Thyroid Association statement on remote-access thyroid surgery. Thyroid 2016;26:331-337.

4 Rogers EM: Diffusion of Innovations, ed 5. New York, Free Press, 2003

5 Garas G, et al: Is there survival benefit from life-long follow-up after treatment for differentiated thyroid cancer? Int J Surg 2013;11:116-121.

6 Tan CT, Cheah WK, Delbridge L: "Scarless" (in the neck) endoscopic thyroidectomy (SET): an evidence-based review of published techniques. World J Surg 2008;32:1349-1357.

7 Christakis I, et al: Minimally invasive endocrine (thyroid, parathyroid, adrenal) surgery: evolution of operative techniques, safety, effectiveness and outcomes; in Hawthorne FT (ed): Minimally Invasive Surgery: Evolution of Operative Techniques, Safety and Effectiveness and Long-Term Clinical Outcomes. New York, Nova Science Publishers, 2014, pp 1-66.

- 8 Chung YS, et al: Endoscopic thyroidectomy for thyroid malignancies: comparison with conventional open thyroidectomy. World J Surg 2007;31:2302-2306; discussion 2307-2308.

9 Moorthy K, et al: Dexterity enhancement with robotic surgery. Surg Endosc 2004;18:790-795.

$\checkmark 10$ Kang SW, et al: Robot-assisted endoscopic surgery for thyroid cancer: experience with the first 100 patients. Surg Endosc 2009;23:2399-2406.

11 Lee SG, et al: Long-term oncologic outcome of robotic versus open total thyroidectomy in PTC: a case-matched retrospective study. Surg Endosc 2016;30:3474-3479.

12 Tae K, et al: Comparison of surgical completeness between robotic total thyroidectomy versus open thyroidectomy. Laryngoscope 2014;124:1042-1047.

13 Tae K, et al: Oncologic outcomes of robotic thyroidectomy: 5-year experience with propensity score matching. Surg Endosc 2016;30:4785-4792.

14 Garas G, et al: Evidence-based surgery: barriers, solutions, and the role of evidence synthesis. World J Surg 2012;36:1723-1731.

15 Aidan P, Chung WY, Lorincz BB: Bilateral vagal automatic periodic stimulation in single-incision transaxillary robotic total thyroidectomy. Clin Otolaryngol 2018;43:401-403.

16 Jackson NR, et al: Safety of robotic thyroidectomy approaches: meta-analysis and systematic review. Head Neck 2014;36:137-143.

17 Materazzi G, et al: Cosmetic result and overall satisfaction after minimally invasive video-assisted thyroidectomy (MIVAT) versus robot-assisted transaxillary thyroidectomy (RATT): a prospective randomized study. World J Surg 2014;38:1282-1288.

18 Chai YJ, et al: A comparative study of postoperative pain for open thyroidectomy versus bilateral axillo-breast approach robotic thyroidectomy using a self-reporting application for iPad. Ann Surg Treat Res 2016;90: 239-245.

19 Ryu HR, et al: A comparison of postoperative pain after conventional open thyroidectomy and transaxillary single-incision robotic thyroidectomy: a prospective study. Ann Surg Oncol 2013;20:2279-2284.

20 Tae K, et al: Robotic thyroidectomy by a gasless unilateral axillo-breast or axillary approach: our early experiences. Surg Endosc 2011;25:221-228.

21 Kandil E, et al: Robotic thyroidectomy versus nonrobotic approaches: a meta-analysis examining surgical outcomes. Surg Innov 2016;23:317-325.

-22 Shen H, Shan C, Qiu M: Systematic review and meta-analysis of transaxillary robotic thyroidectomy versus open thyroidectomy. Surg Laparosc Endosc Percutan Tech 2014;24:199-206.

23 Son SK, et al: Surgical safety and oncologic effectiveness in robotic versus conventional open thyroidectomy in thyroid cancer: a systematic review and meta-analysis. Ann Surg Oncol 2015;22:3022-3032.

-24 Sun GH, Peress L, Pynnonen MA: Systematic review and meta-analysis of robotic vs conventional thyroidectomy approaches for thyroid disease. Otolaryngol Head Neck Surg 2014;150:520-532.

-25 Liu SY, Ng EK: Robotic versus open thyroidectomy for differentiated thyroid cancer: an evidence-based review. Int J Endocrinol 2016;2016:4309087. 
Aidan et al.: Robotic Thyroid Surgery

26 Rabinovics N, Aidan P: Robotic transaxillary thyroid surgery. Gland Surg 2015;4:397-402.

27 Lee J, et al: Perioperative clinical outcomes after robotic thyroidectomy for thyroid carcinoma: a multicenter study. Surg Endosc 2011;25:906-912.

$\checkmark 28$ Arora A, et al: The perception of scar cosmesis following thyroid and parathyroid surgery: a prospective cohort study. Int J Surg 2016;25:38-43.

29 Kim WW, et al: Comparison of the quality of life for thyroid cancer survivors who had open versus robotic thyroidectomy. J Laparoendosc Adv Surg Tech 2016;26:618-624.

30 Linos D, Kiriakopoulos A, Petralias A: Patient attitudes toward transaxillary robot-assisted thyroidectomy. World J Surg 2013;37:1959-1965.

31 Garas G, et al: Is robotic parathyroidectomy a feasible and safe alternative to targeted open parathyroidectomy for the treatment of primary hyperparathyroidism? Int J Surg 2015;15:55-60.

-32 Liu SY, Lang BH: Revisiting robotic approaches to endocrine neoplasia: do the data support their continued use? Curr Opin Oncol 2016;28:26-36.

-33 Chung WY: Pros of robotic transaxillary thyroid surgery: its impact on cancer control and surgical quality. Thyroid 2012;22:986-987.

34 Tolley N, et al: Robotic-assisted parathyroidectomy: a feasibility study. Otolaryngol Head Neck Surg 2011;144: 859-866.

-35 Tolley N, et al: Long-term prospective evaluation comparing robotic parathyroidectomy with minimally invasive open parathyroidectomy for primary hyperparathyroidism. Head Neck 2016;38:E300-E306.

-36 Dralle H: Robot-assisted transaxillary thyroid surgery: as safe as conventional-access thyroid surgery? Eur Thyroid J 2013;2:71-75.

-37 Luginbuhl A, et al: Detection of evolving injury to the brachial plexus during transaxillary robotic thyroidectomy. Laryngoscope 2012;122:110-115.

38 Cabot JC, et al: Robotic and endoscopic transaxillary thyroidectomies may be cost prohibitive when compared to standard cervical thyroidectomy: a cost analysis. Surgery 2012;152:1016-1024.

-39 Trehan A, Dunn TJ: The robotic surgery monopoly is a poor deal. BMJ 2013;347:f7470.

40 Garas G, Arora A, Tolley N: Robotic surgery of the parathyroid glands; in Grillone G, Jalisi S (eds): Robotic Surgery of the Head and Neck. New York, Springer, 2015, pp 133-146.

41 Broome JT, Pomeroy S, Solorzano CC: Expense of robotic thyroidectomy: a cost analysis at a single institution. Arch Surg 2012;147:1102-1106.

42 Wong KA, et al: How can cardiothoracic and vascular medical devices stay in the market? Interact Cardiovasc Thorac Surg 2016;23:940-948.

43 Garas G, et al: Network analysis of surgical innovation: measuring value and the virality of diffusion in robotic surgery. PLoS One 2017;12:e0183332.

44 Drummond MF, et al: Methods for the Economic Evaluation of Health Care Programmes, ed 3. New York, Oxford University Press, 2005.

45 Rao C, Athanasiou T: Cost-effectiveness analysis; in Athanasiou T, et al (eds): Key Topics in Surgical Research and Methodology. London, Springer, 2010, pp 411-420.

46 Kiong KL, et al: Transaxillary thyroidectomies: a comparative learning experience of robotic vs endoscopic thyroidectomies. Otolaryngol Head Neck Surg 2015;152:820-826.

47 Lee J, Chung WY: Robotic surgery for thyroid disease. Eur Thyroid J 2013;2:93-101.

48 Garas G, et al: Which hemostatic device in thyroid surgery? A network meta-analysis of surgical technologies. Thyroid 2013;23:1138-1150.

49 Meltzer C, et al: Surgeon volume in thyroid surgery: surgical efficiency, outcomes, and utilization. Laryngoscope 2016;126:2630-2639.

50 FDA: Medical device class II recall. http://www.fda.gov/ICECI/EnforcementActions/WarningLetters/2013/ ucm363260.htm.

51 Terris DJ, Singer MC, Seybt MW: Robotic facelift thyroidectomy. II. Clinical feasibility and safety. Laryngoscope 2011;121:1636-1641.

52 Richmon JD, Kim HY: Transoral robotic thyroidectomy (TORT): procedures and outcomes. Gland Surg 2017; 6:285-289. 